\title{
Inferior Blow-Out Fracture Reduction Using Two Urinary Balloon Catheters
}

Eun Jun Jo, Jong Hwan Kim, Ho Jik Yang

Department of Plastic and Reconstructive Surgery, Eulji University College of Medicine, Daejeon, Korea

No potential conflict of interest relevant to this article was reported.

\begin{abstract}
Background: The reduction of orbital blowout fracture primarily aims to normalize the extra-ocular movement by returning the herniated orbital soft tissue into the original position, and to prevent enophthalmos by normalizing the orbital cavity volume. We introduce a balloon catheter-assisted orbital floor reduction technique.

Methods: A retrospective review was performed for all patients with orbital floor fracture who underwent the technique described in the main body of this text. Medical records were reviewed for demographic data, clinical presentation and course, degree of enophthalmos, intraorbital volume on computed tomography scan, and postoperative outcomes. The enophthalmos and intraorbital volume of the injured site were compared to the uninjured eye and orbit.

Results: The review identified 14 patients ( 11 male, 3 female). The mean preoperative difference in en-exopthalmos was $2.13 \mathrm{~mm}$, while the mean orbital volume was $116 \%$. The mean postoperative difference in en-exophthalmos had improved to $0.61 \mathrm{~mm}$ with a mean orbital volume of $101.85 \%$. At the time of catheter removal at 10 days, three patients experienced diplopia ( $n=1$ ), extra-ocular movement disorder (1), or enophthalmos (1). All of these had resolved by the 6-month follow-up visit.

Conclusion: Balloon catheter-assisted reduction of the orbital floor fractures was associated with improvements in intraorbital volume and enopthalmos in the 14 patients. Notable complications included diplopia, enophthalmos, and limited extra-ocular movement, all of which were transient in the early postoperative period and had resolved by 6-month follow up.
\end{abstract}

Keywords: Orbital fracture / Blow-out fracture / Urinary catheter

\section{INTRODUCTION}

When the orbital rim experiences blunt impact, the deformation force is transmitted deeper into the orbit and consequently fractures the thin bones of the orbital floor. This results in downward displacement of the intra-orbital muscles and fats into the maxillary sinuses. This is called blowout fracture or orbital floor fracture, which can cause various complications such as enophthal-

Correspondence: Jong Hwan Kim

302-799 Department of Plastic and Reconstructive Surgery, Eulji University

College of Medicine, 95 Dunsanseo-ro, Seo-gu, Daejeon 35233, Korea

E-mail: psjhkim@eulji.ac.kr

Received March 6, 2015 / Revised March 26, 2015 / Accepted March 30, 2015 mos, extra-ocular movement disorder, and diplopia. In the presence of these complications, the fracture site should be accurately evaluated early after the injury through computed tomography (CT) study, and operative reduction should be performed instead of conservative, non-operative treatments [1].

The reduction of blowout fracture aims to return the pushedout intra-orbital materials to their original position and to restore the extra-ocular movement, aside from normalizing the orbital cavity volume to prevent enophthalmosis. Autologous bone graft or artificial grafting materials may be inserted to prevent recurrent herniation of intraorbital soft tissue into the maxillary sinus. Allografts, xenografts, or artificial grafting materials are being used, among which Medpor is often used [2]. However, insertion 
of artificial materials may cause complications such as foreign body reaction, infection, or displaced materials. Autologous bone graft has several disadvantages such as a limited amount of bones harvested, donor site problems, prolonged surgery duration, scars, absorption of the grafted bones, and contouring difficulties. When autologous bone or artificial materials are used to maintain reduced soft tissue, the bone fragments dislocated into the inferior maxillary cavity should either remain there or be completely surgically removed.

Previously, Choi et al. [3] had reported management of orbital floor fracture using endoscopy and a urinary balloon catheter, to prevent maxillary sinusitis, which may be caused by the remaining bony fragment, and to maintain the proper shape of the inferior chamber. As the bony fragment itself is used for reconstruction of the floor, a donor graft is not required. In turn, the disadvantages of using autologous bone are irrelevant in this technique. However, this technique cannot reduce a large fracture and posterior fractures. As such, we have developed a modified technique using two urinary balloon catheters to reduce the floor under direct visualization of the defect through a transconjunctival access.

\section{METHODS}

\section{Subjects and evaluation}

A single-institutional retrospective review was performed for all patients undergoing balloon catheter reduction of blowout fracture. The review identified 14 patients between May 2012 and February 2014. These patients had undergone orbital floor reconstruction using two urinary balloon catheters and were followed up for at least six months. The medical records were examined for demographic information, cause of blowout fracture, physical exam finding, fracture location, presence of concomitant fractures, clinical photographs, and X-ray.

Before the surgery, extra-ocular movement disorder, diplopia, Hertel scale, and the change in the orbital volume in the facial CT results were examined. The Hertel scale is defined as the difference in the distance of the traumatized eye to that of the healthy eye, by measuring from the lateral orbital rim to the apex of the cornea be- tween both sides alternately. The volume of orbital floor fracture was measured by multiplying the inner area of orbital margins on the coronal plane the thickness of CT image slices $(2 \mathrm{~mm})$. The volumes were compared to the uninjured contralateral orbit.

\section{Surgical methods}

Under general anesthesia, limitation of extra-ocular movement was confirmed with forced duction test. A mixture of $1 \%$ lidocain and 1:100,000 epinephrine was injected into the surgical site to minimize the bleeding. The periosteum was exposed via a transconjunctival incision parallel to the orbital margin. The orbital floor was dissected to expose the fracture line.

Using a Caldwell-Luc approach, a 2-cm incision on the mucous membrane was made in the canine fossa of the anterior wall of maxillary sinus to the exposed maxillary sinus. An electric perforator was used to drill two $5 \mathrm{~mm}$ holes into the maxillary sinus, and two urinary balloon catheters were inserted into the floor of the nostrils (Fig. 1). The 12 gauge Foley catheters were expanded with an average of $12 \mathrm{~mL}$ and $8 \mathrm{~mL}$ of normal saline in the anterior and posterior areas, respectively. The degree of enophthalmos was observed after injection of normal saline, and the extra-ocular movement was tested.

The wound was closed using 5-0 Vicryl for the periosteum and 6-0 Vicyl for the conjunctiva. The maxillary sinus incision was closed using 4-0 Vicryl. The two catheters were maintained in

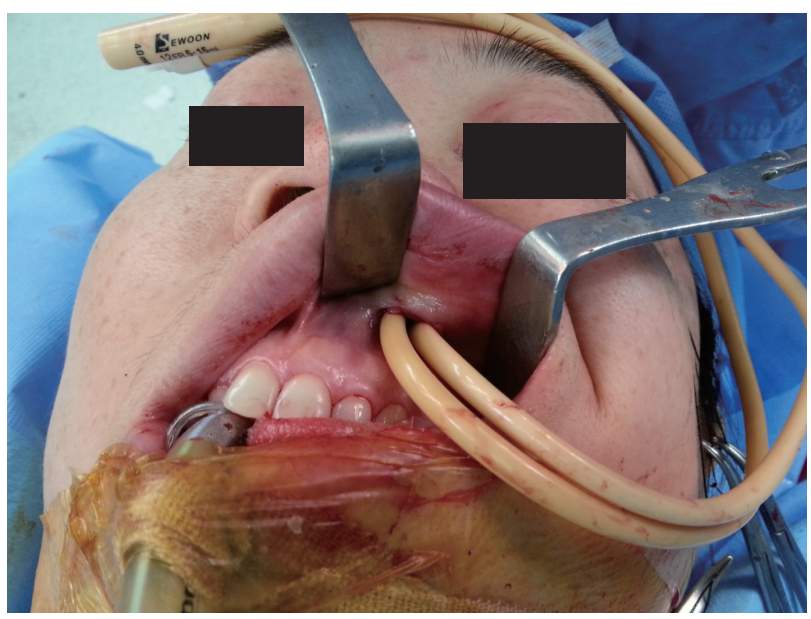

Fig. 1. Surgical field with two urinary balloon catheters inserted. 
place for 10 postoperative days.

\section{RESULTS}

Of the 14 patients, eleven were male and three were female. The fractures were primarily due to motor vehicle collisions, followed by falls, physical altercation, and sports injury (Table 1). Twelve patients had diplopia, 10 had extra-ocular movement disorder. Twelve of the patients had 2-mm enophthalmos prior to operation. Orbital floor fractures were reduced at an average of eight days from the accident.

The mean operation time was 117 minutes. The mean preoperative difference in en-exopthalmos was $2.13 \mathrm{~mm}$, while the mean orbital volume was $116 \%$. The mean postoperative difference in en-exophthalmos had improved to $0.61 \mathrm{~mm}$ with a mean orbital volume of $101.85 \%$ (Table 2). Since no artificial grafts were used, none of the patients required re-operation for a prolapsed grafted material, foreign body reaction, or infection. At the time of catheter removal at 10 days, three patients experienced diplopia $(n=1)$, extra-ocular movement disorder (1), or enophthalmos (1) (Table 3). All of these had resolved by the 6-month follow-up visit and CT showed no signs of movement of the bone fragments (Fig. 2).

\section{DISCUSSION}

The orbital floor has special characteristics with its three-dimensional cone shape. With blunt trauma to the orbit, the intra-orbital pressure increases and is transmitted to the posterior area of inferior orbital groove, the thinnest bone on the orbital floor, which results in fracture. The major complications of this fracture are diplopia and enophthalmos. Diplopia is caused by a shift in the vi-

Table 1. Cause of the blowout fracture

\begin{tabular}{ll} 
Cause & Patients \\
\hline Traffic accident & $7(50)$ \\
\hline Fall & $4(28.6)$ \\
Violence & $2(14.3)$ \\
\hline Sports & $1(7.1)$ \\
\hline Total & $14(100)$ \\
\hline
\end{tabular}

Values are presented as number (\%). sual axis of both eyes due to imbalance of the ocular muscles, and may be caused by the prolapse of inferior rectus muscle, inferior oblique muscle, the Lockwood ligament, and the peri-orbita. Damage of the orbital muscles, including that caused by bony fragments, can also cause diplopia. Enophthalmos can be caused by an increased orbital volume, herniated orbital fat into the maxillary cavity, and posterior traction by the inferior rectus muscle. Facial CT is widely used to observe the changes in the orbital volume, and many studies have investigated the relationship between imagebased orbital volume measurements and the degree of enophthalmos. Hwang et al. [4] reported that enophthalmos increases by 0.71 $\mathrm{mm}$ for each cubic centimeter increase in the orbital volume. Whitehouse et al. [5] reported a similar value of $0.8 \mathrm{~mm}$ enophthalmos for each cubic centimeter increase in the orbital volume. Raskin et al. [6] reported that clinically detectable enophthalmos

Table 2. Change in the Hertel scale and the orbital volume before and after the surgery

\begin{tabular}{|c|c|c|c|c|c|}
\hline \multirow{2}{*}{\multicolumn{2}{|c|}{ Patients Age/Sex }} & \multicolumn{2}{|c|}{ Hertel scale (mm) } & \multicolumn{2}{|c|}{$\begin{array}{l}\text { Percentage of the } \\
\text { orbital volume (\%) }\end{array}$} \\
\hline & & \multicolumn{2}{|c|}{ Preoperation Postoperation } & \multicolumn{2}{|c|}{ Preoperation Postoperation } \\
\hline 1 & 43/Male & 1.8 & 0.3 & 114.6 & 100.6 \\
\hline 2 & 56/Male & 1.9 & 0.1 & 112.5 & 100.4 \\
\hline 3 & 13/Male & 1.7 & 0.8 & 109 & 102.7 \\
\hline 4 & 30/Female & 2.4 & 0.7 & 119.6 & 101.1 \\
\hline 5 & 72/Male & 2.4 & 0.9 & 120.2 & 103 \\
\hline 6 & 47/Male & 1.9 & 0.7 & 115.3 & 100.8 \\
\hline 7 & 64/Female & 2.9 & 0.7 & 124.6 & 101.2 \\
\hline 8 & 54/Female & 3 & 1.1 & 120.6 & 105 \\
\hline 9 & 26/Male & 3.3 & 0.9 & 124.8 & 102.4 \\
\hline 10 & 28/Male & 2.2 & 0.9 & 118.4 & 104.4 \\
\hline 11 & 58/Male & 1.6 & 0.3 & 109.1 & 100.7 \\
\hline 12 & 33/Male & 1.9 & 0.6 & 115.1 & 102.4 \\
\hline 13 & 41/Male & 1.7 & 0.3 & 111.8 & 100.7 \\
\hline 14 & 51/Male & 1.5 & 0.2 & 108.1 & 100.5 \\
\hline Mean & - & 2.16 & 0.61 & 115.98 & 101.85 \\
\hline
\end{tabular}

Table 3. Incidence of complications

\begin{tabular}{lcc} 
Variable & Preoperation & Postoperation \\
\hline Enophthalmos & $12(86)$ & $1(7)$ \\
\hline Diplopia & $10(71)$ & $1(7)$ \\
\hline EOM limitation & $13(93)$ & $1(7)$ \\
\hline
\end{tabular}

Values are presented as number (\%). EOM, extraocular muscle. 

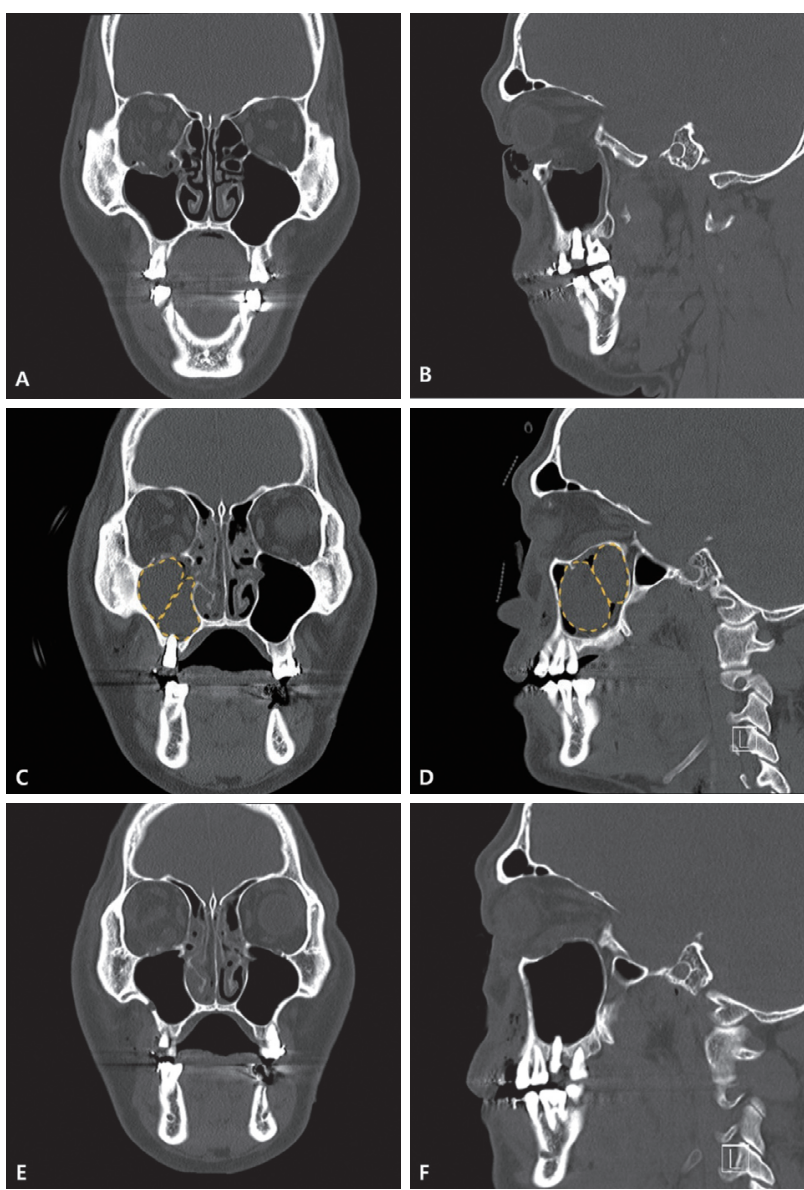

Fig. 2. Facial bone computed tomography (CT) finding of a 43-yearold male with left inferior blow-out fracture. (A) Preoperative coronal CT scan. (B) Preoperative sagittal CT scan. (C) Immediate postoperative coronal CT scan. (D) Immediate postoperative sagittal CT scan. (E) Postoperative coronal CT scan at 6 months. (F) Postoperative sagittal CT scan at 6 months.

occurs with orbital volume increase of $13 \%$ or greater. McGurk et al. [7] reported that the ratio of orbital volume can be measured by comparing the fractured side with the normal side, as most individuals have similar orbital volumes in both sides. Because of these previous findings, we chose to evaluate the pathologic orbital volume as a percentage of the uninjured, contralateral side. This was designed to reduce any error caused by individual differences in the orbital volume. In our patients, we found that the injured orbit had a volume that was $116 \%$ of the orbital volume of the uninjured site, prior to the surgery. This value had changed to a more comparable ratio of $101.8 \%$ after the operation.

Orbital floor fracture with significant prolapsed orbital content on CT imaging should be reconstructed to prevent enophthalmos of the intra-orbital tissues [8,9]. Many surgical methods have been introduced to treat orbital floor fracture. Currently, the most widely used treatment approach is the reconstruction of floor using graft materials through subciliary or transconjunctival access. The graft may be autologous bone or artificial material. An autologous bone graft decreases the risk of infection compared with an artificial grafting material, but is difficult to manipulate into the natural shape of the orbital floor. Donor-site morbidity and graft absorptions are additional concerns. Over-correction is required to prevent the aforementioned disadvantages of the autologous bone graft, but the degree of correction was not investigated in this study to prevent distortion from bone absorption [10,11]. Artificial grafting materials such as Medpor are also widely used [12], but are associated with complications such as infection and increased cost of care. When the orbital floor fracture extends into posterior regions, the graft materials may not be adequately sized to reconstruct the whole of defect.

Bony fragments can also be used for an autograft stented with a urinary balloon catheter. This technique involves the reduction of anatomical structure of orbits by lifting the bone fragments and filling the maxillary cavity through balloon expansion. However, fracture of the posterior orbital floor is difficult to correct using this technique due to the balloon shape. Excessive injection of normal saline for posterior reduction may burst the balloon or overcorrect the fracture. In our study, a transconjunctival incision was used to reduce the herniated content, and the balloons in the maxillary cavity were expanded while the orbital floor was observed through the transconjunctival access.

In our experience, this balloon-assisted splinting under direct visualization of orbital floor was able to maintain proper reduction of the floor while maintaining the concavity. Postoperative facial CT scans were used to assess over/under-correction of the fracture. Depending on the degree, the balloon catheters were further inflated or deflated to further optimize the reduced state. Because no artificial materials were needed for reconstruction, none of the patient underwent re-operation from prolapsed materials, foreign body reaction, or infections.

Postoperative evaluation of floor fracture includes functional 
abnormalities (such as limited extra-ocular movement or diplopia), enophthalmos, and facial CT. Enophthalmos is usually examined using Hertel exophthalmometry. However, this result may not be reliable due to interobserver variability and changes due to edema [13]. In our study, two examiners measured enophthalmos two times in a row, and the mean value was obtained to minimize the observer variability. This was evaluated one day before the surgery to minimize the effect of edema. The postoperative evaluation was carried out at six months, after the bony orbit union and atrophy of recovered fat tissue. The mean enophthalmos deceased from 2.16 to $0.61 \mathrm{~mm}$ after the surgery.

The complication rates in this study were 7\% for diplopia, 7\% for limited extra-ocular movement, and 7\% for enophthalmos. However, these symptoms were resolved at six months after respective operations, and no distortion of orbital floor wall was observed on facial CT scan.

In this study, we characterized 14 subjects who underwent balloon catheter assisted reduction of orbital floor fracture. Operative outcomes were satisfactory for intraorbital volume and enopthalmos. Notable complications included diplopia, enophthalmos, and limited extra-ocular movement, all of which were transient in the early postoperative period and had resolved by 6 -month follow up.

\section{REFERENCES}

1. Saunders CJ, Whetzel TP, Stokes RB, Wong GB, Stevenson TR. Transantral endoscopic orbital floor exploration: a cadaver and clinical study. Plast Reconstr Surg 1997;100:575-81.

2. Joo K, Chung SH, Han KT, Kwon H, Im JS, Kang YJ. A comparative study of the implants used in the management of blowout fracture. J Korean Soc Plast Reconstr Surg 1999;26:470-6.

3. Choi HJ, Kim MS, Lee YM. The endoscopic treatment of blow-out fracture. J Korean Cleft Palate-Craniofac Assoc 2002;3:173-8.

4. Hwang WB, Bae YC, Jeon JY, Hwang SM, Lee J, Kim DH. Orbital volume change in post-traumatic enophthalmos. J Korean Soc Plast Reconstr Surg 1997;24:1031-43.

5. Whitehouse RW, Batterbury M, Jackson A, Noble JL. Prediction of enophthalmos by computed tomography after 'blow out' orbital fracture. Br J Ophthalmol 1994;78:618-20.

6. Raskin EM, Millman AL, Lubkin V, della Rocca RC, Lisman RD, Maher EA. Prediction of late enophthalmos by volumetric analysis of orbital fractures. Ophthal Plast Reconstr Surg 1998;14:19-26.

7. McGurk M, Whitehouse RW, Taylor PM, Swinson B. Orbital volume measured by a low-dose CT scanning technique. Dentomaxillofac Radiol 1992;21:70-2.

8. Grant MP, Iliff NT, Manson PN. Strategies for the treatment of enophthalmos. Clin Plast Surg 1997;24:539-50.

9. Kim YH, Seul JH. An analysis of delayed correction of 25-cases of post traumatic ocular displacement. J Korean Soc Plast Reconstr Surg 1997;24:1016-30.

10. Johnson PE, Raftopoulos I. In situ splitting of a rib graft for reconstruction of the orbital floor. Plast Reconstr Surg 1999;103:1709-11.

11. Kline RM Jr, Wolfe SA. Complications associated with the harvesting of cranial bone grafts. Plast Reconstr Surg 1995;95:5-13.

12. Jobe R, Iverson R, Vistnes L. Bone deformation beneath alloplastic implants. Plast Reconstr Surg 1973;51:169-75.

13. Musch DC, Frueh BR, Landis JR. The reliability of Hertel exophthalmometry: observer variation between physician and lay readers. Ophthalmology 1985;92:1177-80. 